\title{
Excited state modulation in Donor-Substituted Multi-resonant Thermally Activated Delayed Fluorescence Emitters
}

Sen Wu, ${ }^{a}$ Wenbo Li, ${ }^{b}$ Kou Yoshida, ${ }^{b}$ David Hall,,${ }^{a, c}$ Subeesh Madayanad Suresh, ${ }^{a}$ Thomas Sayner, ${ }^{b}$ Junyi Gong, ${ }^{b}$ David Beljonne, ${ }^{c}$ Yoann Olivier, ${ }^{*}$ Ifor D. W. Samuel ${ }^{b *}$ and Eli Zysman-Colman ${ }^{a^{*}}$

${ }^{a}$ Organic Semiconductor Centre, EaStCHEM School of Chemistry, University of St Andrews, St Andrews, Fife, UK, KY16 9ST, Fax: +44-1334 463808; Tel: +44-1334 463826; E-mail: eli.zysman-colman@st-andrews.ac.uk.

${ }^{\text {b}}$ Organic Semiconductor Centre, SUPA School of Physics and Astronomy, University of St Andrews, St Andrews, UK, KY16 9SS; E-mail: idws@st-andrews.ac.uk

${ }^{c}$ Laboratory for Chemistry of Novel Materials, University of Mons, 7000, Mons, Belgium.

${ }^{\mathrm{d}}$ Laboratory for Computational Modeling of Functional Materials \& Solid State Physics Laboratory, Namur Institute of Structured Matter, University of Namur, Rue de Bruxelles, 61, 5000 Namur, Belgium. E-mail: yoann.olivier@unamur.be

\section{Abstract}

Examples of multi-resonant thermally activated delayed fluorescence (MR-TADF) emitters that emit at longer wavelengths remain rare. To reach that goal, we decorate different numbers and types of electron-donors about a central MR-TADF core, DiKTa. Depending on the identity and number of donor groups, the excited state either remains short range charge transfer (SRCT) and thus characteristic of an MR-TADF emitter or becomes a long-range charge transfer (CT) that is typically observed in donor-acceptor TADF emitters. The impact is that in three examples, Cz-DiKTa, Cz-Ph-DiKTa and $\mathbf{3 C z}$-DiKTa, which emit from a SRCT state, the emission remains narrow, while in four examples, TMCz-DiKTa, DMACDiKTa, 3TMCz-DiKTa and 3DMAC-DiKTa, which emit via a CT state, the emission broadens significantly. Through this strategy, the organic light-emitting diodes fabricated with the three MR-TADF emitters show maximum electroluminescence emission wavelengths, $\lambda_{\mathrm{EL}}$, of 511, 492 and $547 \mathrm{~nm}$ with moderate full-width at half maxima (FWHM) of 62, 61 and 54 $\mathrm{nm}$, respectively. Importantly, each of these devices show high maximum external quantum efficiencies $\left(\mathrm{EQE}_{\max }\right)$ of $24.4 \%, 23.0 \%$ and $24.4 \%$, which are amongst the highest reported 
with ketone-based MR-TADF emitters. OLEDs with D-A type emitters, DMAC-DiKTa and TMCz-DiKTa, also show high efficiencies, with $\mathrm{EQE}_{\max }$ of $23.8 \%$ and $20.2 \%$, but accompanied by broad emission at $\lambda_{\mathrm{EL}}$ of 549 and $527 \mathrm{~nm}$, respectively. Noteworthy is that the DMAC-DiKTa-based OLED shows very small efficiency roll-off, and its EQE remains 18.5\% at $1000 \mathrm{~cd} \mathrm{~m}^{-2}$. Therefore, this work demonstrates that manipulating the nature and numbers of donor groups decorating a central MR-TADF core is a promising strategy for both red-shifting the emission and improving the performance of the OLEDs.

\section{Introduction}

Thermally activated delayed fluorescence (TADF) materials are a promising class of emitters for organic light-emitting diodes (OLEDs) as the devices can realise up to $100 \%$ internal quantum efficiency while the organic emitters can be easily synthesized at low cost and are sustainable. ${ }^{1-3}$ TADF operates by converting non-emissive triplet excitons into singlets through endothermic reverse intersystem crossing (RISC). RISC is typically only possible when there is spin-orbit coupling (SOC) between the triplet and singlet states, and the energy gap between them $\left(\Delta E_{\mathrm{ST}}\right)$ is small. The magnitude of $\Delta \mathrm{E}_{\mathrm{ST}}$ is correlated with the degree of overlap of the orbitals involved in the transition, which typically are the highest occupied molecular orbital (HOMO) and lowest unoccupied molecular orbital (LUMO). ${ }^{4}$ The corresponding molecular design usually involves minimizing the conjugation between the electron-donating moieties and electron-accepting moieties by adopting a strongly twisted conformation between these two units. This commonly used design has a number of drawbacks. Due to the large redistribution of the electron density during the transition, the nature of the emission is charge transfer (CT) between the donor and acceptor. ${ }^{5}$ This, coupled with the conformational flexibility inherent in the design, leads to a broad emission spectrum (full width half-maximum, FWHM, of 80-200 nm). ${ }^{6}$ This significantly degrades the color gamut of the OLEDs, which is 
an undesirable trait for displays. Therefore, there is at present a growing effort to develop TADF materials that show both a small $\Delta E_{\mathrm{ST}}$ and narrow emission spectra.

One subclass of TADF emitters that responds to these criteria are multi-resonant TADF emitters (MR-TADF). Examples of MR-TADF emitters are shown in Figure S3. MR-TADF emitters are nanographenes containing suitably positioned electron-donating atoms (e.g., N, O, S) and electron-deficient atoms or groups (e.g., B, C=O) within the fused aromatic framework. In these compounds, electrons and holes are localized on adjacent atoms due to the complementary mesomeric effect of the electron-donating and electron-accepting units leading to the required small exchange integral and $\Delta E_{\mathrm{ST} .}{ }^{7}$ Although nearly $100 \mathrm{MR}$-TADF emitters have been reported since the first example in $2016,{ }^{8}$ the majority of these show blue or green emission. There are still very few examples that emit at longer wavelengths. ${ }^{7}$ Therefore, this work focusses on developing longer wavelength MR-TADF emitters.

There are several potential strategies that may be employed to tune the emission energy towards the red. The first involves strategic placement of the relative positions of the electron-donating and electron-accepting groups. In 2020, Yasuda and co-workers, ${ }^{9}$ arranged two electrondonating nitrogen atoms para to each other and two boron atoms para to each other about the central phenyl ring (namely $\mathrm{B}-\pi-\mathrm{B}$ and $\mathrm{N}-\pi-\mathrm{N}$ ), which led to a significantly red-shifted emission and is the first example of a red MR-TADF emitter, BBCz-R, showing an emission maximum, $\lambda_{\mathrm{PL}}$, of $615 \mathrm{~nm}$ in toluene solution. Duan and co-workers, ${ }^{10}$ adopted a similar strategy, producing two red emitters, R-BN and R-TBN, with $N-\pi-\mathrm{N}$ arrangements $\left(\lambda_{\mathrm{PL}}=662\right.$ and $692 \mathrm{~nm}$ in toluene solution). A second strategy involves extending the conjugation length of the MR-TADF emitter core. Hatakeyama and co-workers, ${ }^{11}$ reported a green emitter $\left(\lambda_{\mathrm{PL}}\right.$ of $506 \mathrm{~nm}$ in $1 \mathrm{wt} \%$ PMMA film), OAB-ABP-1, that contains an extended $\pi$-skeleton that consists of an alternating pattern of para-disposed O-B-N atoms. Kido and co-workers ${ }^{12}$ reported a 
green emitter PXZ-BN with $\lambda_{\text {PL }}$ of $502 \mathrm{~nm}$ by replacement of carbazole for phenoxazine within BCz-BN skeleton. In a similar vein, Yang and co-workers incorporated sulfur, affording the green emitter 2PTZBN $\left(\lambda_{\mathrm{PL}}=510 \mathrm{~nm}\right.$ in toluene $)$, with the expectation of enhancing spin-orbit coupling and hence the reverse intersystem crossing rate. ${ }^{13} \mathrm{~A}$ third strategy to modulate the emission involves the incorporation of peripheral electron-donating or electron-accepting moieties. Duan and co-workers, ${ }^{14}$ reported the first examples of green-emitting MR-TADF emitters $\left(\lambda_{\mathrm{PL}}=502 \mathrm{~nm}\right.$ in $6 \mathrm{wt} \%$ doped mCPCB film), $\mathbf{2 F - B N}$, by decorating peripheral electron-withdrawing fluorophenyl groups para to the central boron atom. Wang and coworkers, also employed the same strategy, incorporating electron-withdrawing benzonitrile units in the compound (R)-OBN-4CN-BN, obtaining a green emitter $\left(\lambda_{\mathrm{PL}}=500 \mathrm{~nm}\right.$ in toluene $)$. Duan and co-workers, ${ }^{15}$ reported another green-emitting MR-TADF compound (AZA-BN) that incorporates a fused azaphenanthrene $\left(\lambda_{\mathrm{PL}}=522 \mathrm{~nm}\right.$ in toluene). Using the same $\mathbf{B C z}-\mathbf{B N}$ skeleton, Wang and co-workers, reported the green emitter $(\boldsymbol{m}-\mathbf{C z}-\mathbf{B N C z})$ that contains a metadisposed auxiliary di-tert-butylcarbazole with respect to the central boron atom $\left(\lambda_{\mathrm{PL}}=519 \mathrm{~nm}\right.$ in toluene). ${ }^{16}$ Recently, Yang and co-workers, introduced donor groups at the para position of the carbazole of the $\mathbf{B C z}-\mathbf{B N}$ skeleton and demonstrated color modulation from sky blue to yellow ( $\lambda_{\mathrm{PL}}$ of 496 to 562 in toluene) ${ }^{17}$ Using the same BCz-BN skeleton, You and co-workers, combined both donor and acceptor groups located para to the $\mathrm{N}$ and $\mathrm{B}$ atoms, respectively, to realize orange emission $\left(\lambda_{\mathrm{PL}}\right.$ of $581 \mathrm{~nm}$ in toluene). ${ }^{18}$ Although $\mathrm{B} / \mathrm{N}$-based emitters have realized full-colour emission, their synthesis can only be reached through lithiationborylation-cyclization reaction or electrophilic fixed-point $\mathrm{C}-\mathrm{H}$ borylation cyclization reaction, which complicates downstream elaboration of these structures. A second family of MR-TADF compounds employ electron-accepting carbonyl groups in lieu of boron atoms. We showed that decorating the MR-TADF DiKTa with mesityl groups, Mes 3 DiKTa, can mitigate undesired aggregation caused quenching (ACQ) and excimer emission while also modestly 
red-shifting the emission $\left(\lambda_{\mathrm{PL}}=468 \mathrm{~nm}\right.$ in toluene $) .{ }^{19} \mathrm{We}$ also reported a dimeric compound, DDiKTa, consisting of two DiKTa units, that showed a red-shifted emission with $\lambda_{\text {PL }}$ of 500 nm. ${ }^{20}$ Liao and co-workers, reported structurally rigid analogs of DiKTa that incorporated a carbon- oxygen- or sulfur-based tether. The compounds DQAO, OQAO and SQAO showed red-shifted emission compared to DiKTa with $\lambda_{\mathrm{PL}}$ ranging from 465 to $552 \mathrm{~nm}$ in toluene. ${ }^{21}$ Zhang and co-workers, reported the compounds QAD-Cz, QAD-2Cz and QAD-mTPDA that contain donor groups decorating the DiKTa core to afford D-A type emitters. These molecules showed blue to red emission with $\lambda_{\mathrm{PL}}$ ranging 488 to $586 \mathrm{~nm}$ in toluene solution. ${ }^{22}$

In this context, we decorated the DiKTa core with different numbers of donors with differing electron-donating strengths. These donors include carbazole $(\mathrm{Cz})$, 9,9-dimethylacridan (DMAC), carbazolyl-phenyl (Cz-Ph) and 1,3,6,8-tetramethyl-9H-carbazole (TMCz). These donors were positioned para to the central electron-donating nitrogen atom. We thus built a framework to systematically study the impact on the emission colour and nature of the excited state of the inclusion of these electron-donating groups (Figure 1). It was found that by introducing weak donors such as carbazole to the para-carbon position of nitrogen, the HOMO levels of the new emitters were destabilized significantly compared to that of the parent DiKTa, while the LUMO levels were barely perturbed, leading to the desired red-shifted emission. Importantly, the narrow emission characteristic of MR-TADF emitters was maintained. However, when the electron-donating strength was further increased, the emission nature changed to $\mathrm{CT}$ and the emission spectra significantly broadened. In this study, weaker-donorbased emitters, Cz-DiKTa, 3Cz-DiKTa and Cz-Ph-DiKTa maintained their MR-TADF character in all the tested environments, while, stronger-donor-based emitters, TMCz-DiKTa, DMAC-DiKTa, 3TMCz-DiKTa and 3DMAC-DiKTa showed a more complicated behavior where CT emission dominates in polar media. 


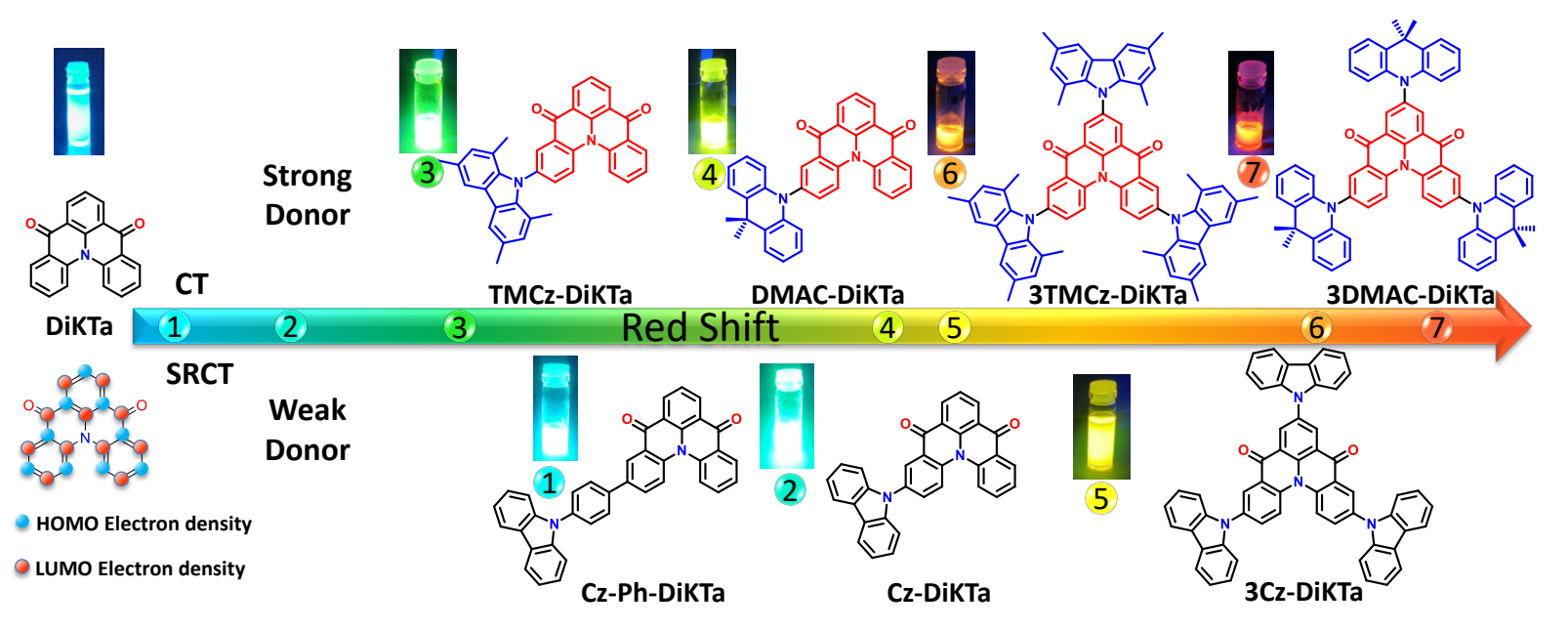

Figure 1. The molecular design of DiKTa-based MR-TADF emitters and their emission color. The compounds above the arrow contain stronger donors and emit via a long-range CT state and show broad emission while the compounds below the arrow contain weaker donors and emit via a SRCT state and show narrowband emission. The images are toluene solutions photoexcited at $365 \mathrm{~nm}$.

\section{Results and Discussion}

\section{Synthesis}

Seven donor-substituted DiKTa emitters, Cz-DiKTa, Cz-Ph-DiKTa, TMCz-DiKTa, DMAC-DiKTa, 3Cz-DiKTa, 3TMCz-DiKTa and 3DMAC-DiKTa, were obtained following either palladium-catalyzed Buchwald-Hartwig amination or Suzuki-Miyaura cross-coupling with suitably brominated DiKTa intermediates as outlined in Scheme S1. The identity and purity of the seven emitters were ascertained using a combination of ${ }^{1} \mathrm{H}$ NMR and ${ }^{13} \mathrm{C}$ NMR spectroscopy, HRMS, HPLC and element analysis, and melting point determination.

\section{Theoretical Studies}

The frontier molecular orbitals (FMOs) of these emitters were firstly modelled based on the optimized ground state gas-phase geometry using Density Functional Theory (DFT) at the PBE0/6-31G(d,p) level of theory. The HOMO and LUMO distributions are shown in Figure S5 and the HOMO and LUMO level of the seven compounds are listed in the Table S1. 
Compared to DiKTa (-5.94/-2.31 eV), the HOMO level is destabilized by 0.21 to $0.68 \mathrm{eV}$, and the degree of destabilization correlates with both the strength and numbers of the peripheral donor group. We noticed that the occupied orbital localized on the DiKTa core of the series of donor-substituted DiKTa compounds does not correspond to the HOMO, instead residing on the donor (see Figure S5). For the compounds with the weakest donors (Cz-DiKTa, Cz-PhDiKTa, 3Cz-DiKTa) this orbital slightly delocalizes to the carbazoles, thereby resulting in destabilization compared to DiKTa while for the compounds containing the strongest donors (TMCz-DiKTa, DMAC-DiKTa, 3DMCz-DiKTa) the energy of the DiKTa-localized orbital remains unaffected.

We then employed the Tamm-Dancoff approximation to time-dependent DFT (TDA-DFT) to predict the energies of the excited states (Table S1). ${ }^{23-24}$ TDA-DFT calculations predict that the nature of the lowest singlet excited state $\left(\mathrm{S}_{1}\right)$ of Cz-DiKTa, 3Cz-DiKTa, Cz-Ph-DiKTa, TMCz-DiKTa, DMAC-DiKTa, 3TMCz-DiKTa and 3DMAC-DiKTa is CT that is characteristic of D-A type TADF compounds as shown in Figure S5. ${ }^{19}$ However, we have previously demonstrated that it is essential to use suitably high-level theory models to accurately predict the excited state energies of MR-TADF compounds. ${ }^{6}$ Therefore, we employed Spin-Component Scaling Coupled-Cluster second-order approximate CoupledCluster (SCS-CC2) with the cc-pVDZ basis set to more accurately model the nature of the charge transfer excited states. Figure 2 shows the difference density plots of the $S_{1}$ and the second lowest singlet excited state $\left(\mathrm{S}_{2}\right)$ transitions of these emitters obtained using SCS-CC2. Cz-DiKTa, Cz-Ph-DiKTa and 3Cz-DiKTa all exhibit similar $\mathrm{S}_{1}$ difference density plot patterns akin to that for DiKTa (shown in Figure S7). ${ }^{19}$ Based on the charge-transfer distance, $\mathrm{D}_{\mathrm{CT}}<1.4 \AA$, we assign these excited states to be SRCT (Table S2). At the same time, small contributions to the difference density plots can be seen at the peripheral donor fragments in the new emitters, especially on the "top" carbazole moiety in 3Cz-DiKTa. The $\mathrm{S}_{2}$ state of each 
of Cz-DiKTa, TMCz-DiKTa and Cz-Ph-DiKTa possesses $n-\pi^{*}$ character, while for $\mathbf{3 C z}-$ DiKTa $\mathrm{S}_{2}$ remains a $\pi-\pi^{*}$ transition. The $\mathrm{S}_{1}$ difference density plot of DMAC-DiKTa is almost identical to that of DiKTa, while the $\mathrm{S}_{2}$ difference density plots shows a D-A type long range CT character as the increased density can be seen on the electron-deficient DiKTa core and the decreased density is located on the peripheral DMAC moieties. Indeed, the $\mathrm{D}_{\mathrm{CT}}$ of this state is $3.18 \AA$, characteristic of a long-range CT state. Since all the calculations are carried out in gasphase, potentially the nature of the emissive excited state may switch between MR-TADF CT and D-A CT, depending on the environment as the energy gap between the $\mathrm{S}_{1}$ and $\mathrm{S}_{2}\left(\Delta E_{\mathrm{S} 1 \mathrm{~S} 2}\right)$ of this emitter is small $(0.34 \mathrm{eV})$ and the $\mathrm{S}_{2}$ electrical dipole moment for the $\mathrm{S}_{2}$ state is large. We also performed the same calculations on some reported donor-acceptor type emitters containing an MR-TADF moiety acting as the acceptor, to validate our computational methodology (Figure S4). ${ }^{25}$ All the investigated emitters show SRCT $\mathrm{S}_{1}$ states in the gas phase calculations and a narrow emission characteristic of a MR-TADF behavior. ADBAN-MeMesCz and DABNA-2 show large $\Delta E_{\mathrm{S} 1 \mathrm{~S} 2}$ of 0.71 and $0.64 \mathrm{eV}$ at the SCS-CC2 level, which suggests that this $\Delta E_{\mathrm{S} 1 \mathrm{~S} 2}$ energy gap is large enough to prevent the switching of light emission from the SRCT state to the long-range CT state when considering the impact of solvent effects or polarization effects arising in the solid state. By contrast, PXZ-DABOA, TDBAAc, TDBADI and QAO-dic all have predicted D-A type CT $\mathrm{S}_{2}$ states, and much smaller $\Delta E_{\mathrm{S} 1 \mathrm{~S} 2}$ values of 0.04-0.29 eV. Such a small energy difference implies that a state inversion becomes possible when the medium is sufficiently polar. When considering 3DMCz-DiKTa and 3DMACDiKTa, the $\mathrm{S}_{1}$ state shows a strong contribution of the hole density on the top $\mathrm{Cz}$ unit while the electron density is mainly localized on the DiKTa core, resulting in a long-range CT state. The calculated $\mathrm{S}_{1} / \mathrm{T}_{1}$ energies for the MR-TADF predicted emitters, Cz-DiKTa, Cz-PhDiKTa and 3Cz-DiKTa, are 3.35/3.09, 3.39/3.13 and 3.05/2.81 eV, respectively, with corresponding $\Delta E_{\mathrm{ST}}$ values of $0.26,0.26$ and $0.24 \mathrm{eV}$. These $\Delta E_{\mathrm{ST}}$ values are of similar 
magnitude to that of DiKTa $(0.27 \mathrm{eV})$, and it indicates a likely similar TADF efficiency in these compounds. As 3TMCz-DiKTa and 3DMAC-DiKTa are predicted to be D-A dominant TADF emitters, the $\Delta E_{\mathrm{ST}}$ obtained from the SCS-CC2 calculations are smaller ( 0.18 and 0.03 $\mathrm{eV}$, respectively).

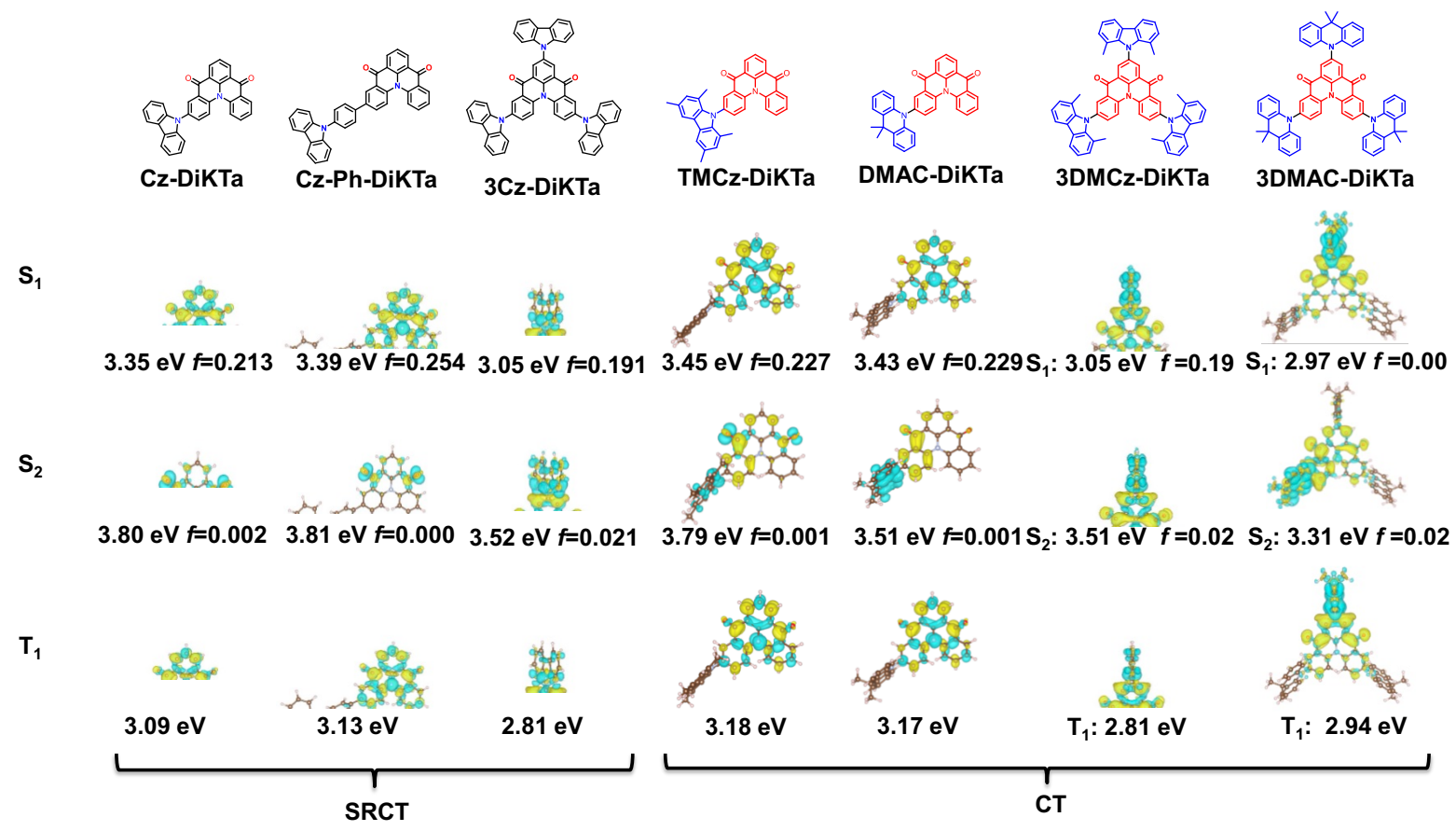

Figure 2. (a) Molecular structures and difference density plots of $S_{1}$ and $S_{2}$ excited states (calculated in the gas phase at the SCS-CC2/cc-pVDZ level) for Cz-DiKTa, Cz-Ph-DiKTa, 3Cz-DiKTa, TMCz-DiKTa, DMAC-DiKTa, 3DMCz-DiKTa and 3DMAC-DiKTa, $f$ is the oscillator strength.

\subsection{Optoelectronic Properties}

Cyclic voltammetry (CV) and differential pulse voltammetry (DPV) measurements were used to experimentally determine HOMO and LUMO levels. The CV and DPV profiles in dichloromethane are shown in Figure 3a (3TMCz-DikTa and 3DMAC-Dikta are shown in Figure S8) and the electrochemical data are summarized in Table S3. The CV profile of $\mathbf{C z}-$ DiKTa, Cz-Ph-DiKTa, TMCz-DiKTa, DMAC-DiKTa, 3Cz-DiKTa, 3TMCz-DiKTa and 3DMAC-DiKTa all show reversible reduction waves, which corresponds to the reduction localized on the DiKTa core. While Cz-DiKTa and Cz-Ph-DiKTa show irreversible oxidation 
waves, which are assigned to the oxidation of the carbazole, when the donor is DMAC and $\mathrm{TMCz}$, the oxidation waves become significantly more reversible. The $E_{\text {red }} / E_{\text {ox }}$ values of all seven emitters are determined from the peak of the DPVs. The LUMO levels of Cz-DiKTa, Cz-Ph-DiKTa, TMCz-DiKTa and DMAC-DiKTa are almost identical to that of DiKTa (HOMO/LUMO values of -6.12/-3.00). When the numbers of donors are increased (3CzDiKTa, 3TMCz-DiKTa and 3DMAC-DiKTa), the LUMO stabilizes by ca. $0.2 \mathrm{eV}$. In comparison, increasing the electron-donating strength of the peripheral donor (e.g., from $\mathrm{Cz}$ to DMAC) results in shallower HOMO levels. Similarly, increasing the number of electron donors also results in shallower HOMOs. Therefore, both strategies can be used to reduce the HOMO-LUMO band gap.

The room-temperature ultraviolet-visible (UV-Vis) absorption, steady-state photoluminescence (PL), recorded at room-temperature (SS RT), and PL spectra of the prompt and delayed emission recorded at $77 \mathrm{~K}$, (the latter being the phosphorescent spectra) in toluene $\left(10^{-5} \mathrm{M}\right)$ are shown in Figures 3b-f (Figure S9 shows the PL spectra for 3TMCz-DiKTa and 3DMAC-DiKta), the corresponding data are summarized in Table $\mathbf{S 4}$. The absorption spectra all show two characteristic absorption bands. The higher energy bands $(300-430 \mathrm{~nm})$ are attributed to $\pi-\pi^{*}$ locally excited (LE) transitions of both the donors and the DiKTa core, and the lower energy bands between 443-492 $\mathrm{nm}$ are attributed to SRCT transitions centered on the DiKTa core that are characteristic of MR-TADF emitters. Compared to DiKTa, ${ }^{19}$ the lowestenergy absorption band of the Cz-DiKTa, Cz-Ph-DiKTa and 3Cz-DiKTa progressively redshifts and becomes broader and weaker as shown in Figure 3, suggesting increasing CT character of the transitions associated with this band. The CT absorption bands of TMCzDiKTa and DMAc-DiKTa have lower molar absorptivity, which can be rationalized by the small FMO overlap due to the strongly twisted conformation of the bulky TMCz donor and the electron-donating strength of the DMAC donor. The seven compounds show a progressively 
red-shifted emission with increasing number and strength of the electron-donors from 472 to $667 \mathrm{~nm}$ compared with DiKTa $\left(\lambda_{\mathrm{PL}}=460 \mathrm{~nm}, \mathrm{FWHM}=27 \mathrm{~nm}\right)$. Cz-DiKTa, Cz-Ph-DiKTa and 3Cz-DiKTa show small full width half maximum (FWHM) of 54, 47 and $53 \mathrm{~nm}$, respectively. The small FWHM is correlated to the small Stokes shifts of 31-50 nm, which indicates that the structural relaxation is small in their excited states. In contrast, the Stokes shifts of TMCz-DiKTa, DMAC-DiKTa, 3TMCz-DiKTa and 3DMAC-DiKTa are much larger ( 86 to $242 \mathrm{~nm}$ ) and the FWHM are greater than $80 \mathrm{~nm}$. These results indicate that in the presence of strong electron-donating groups, the SRCT character of the excited state disappears and long-range D-A type CT character starts to be dominant. These observations agree with the computational results shown in the Figure 2. We next probed how the nature of the emissive excited state evolves as a function of solvent polarity and the results are presented in Figure S10. Cz-DiKTa, Cz-Ph-DiKTa and 3Cz-DiKTa all show a small degree of positive solvatochromism that is characteristic of SRCT states associated with MR-TADF emitters. ${ }^{19}$ By contrast, TMCz-DiKTa, DMAC-DiKTa, 3TMCz-DiKTa and 3DMAC-DiKTa show significant positive solvatochromism, suggesting the lowest excited states of these compounds, especially in polar media, are CT in nature. They also show CT and SRCT dual emission in high polarity solvents, with emission moving from SRCT to CT with increasing polarity (Figure S10). This was observed previously using a MR-TADF core (ADBNA-Me-Mes), with $\mathrm{NMe}_{2}$ substitution. ${ }^{26}$

The $\Delta E_{\mathrm{ST}}$ values were determined from the difference in energy of the onsets of the prompt fluorescence and phosphorescence spectra in toluene at $77 \mathrm{~K}$. The corresponding $\Delta E_{\mathrm{ST}}$ values of Cz-DiKTa, Cz-Ph-DiKTa, TMCz-DiKTa, DMAC-DiKTa, 3Cz-DiKTa, 3TMCz-DiKTa and 3DMAC-DiKTa are $0.20,0.22,0.23,0.21,0.11,0.01$ and $0.01 \mathrm{eV}$, respectively. The $\Delta E_{\mathrm{ST}}$ values of Cz-DiKTa, Cz-Ph-DiKTa and 3Cz-DiKTa are consistent with the SCS-CC2 calculations, which implies the emissive excited state is SRCT in nature. TMCz-DiKTa shows 
a broader emission spectrum but possesses an identical $\Delta E_{\mathrm{ST}}$ value to the predicted one, which indicates that there may be mixed SRCT/CT character in low polarity solvents such as toluene. The calculated D-A type emitters DMAC-DiKTa, 3TMCz-DiKTa and 3DMAC-DiKTa, show much smaller $\Delta E_{\mathrm{ST}}$ values, which reflects the smaller overlap integral.

(a)

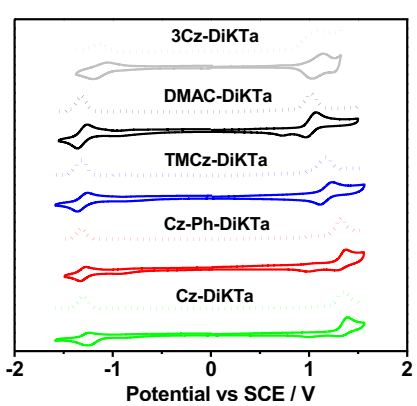

(d)

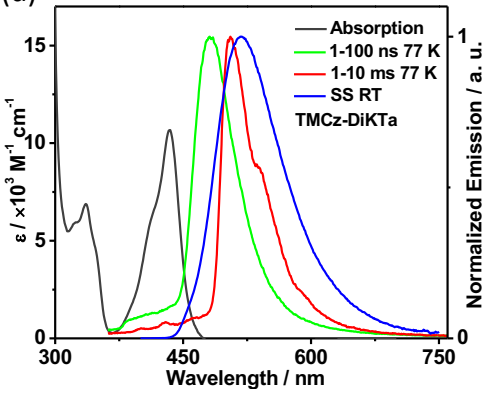

(b)

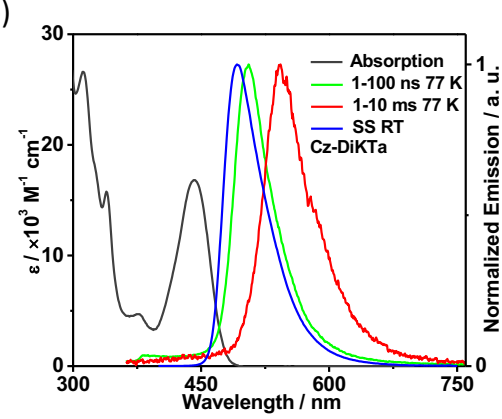

(e)

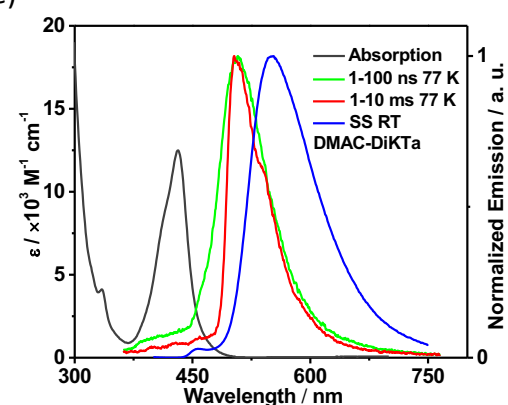

(c)

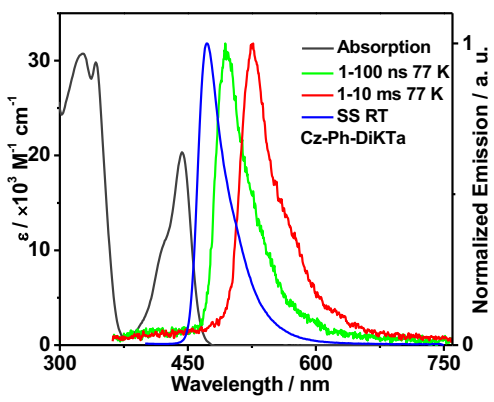

(f)

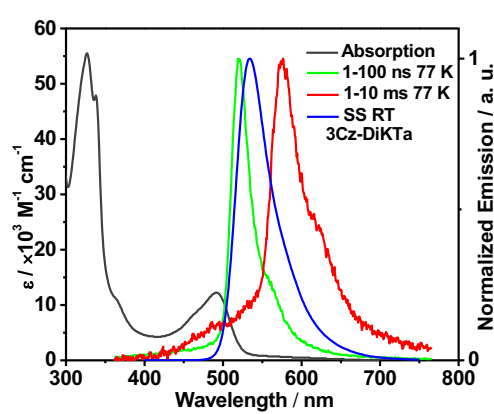

Figure 3. (a) Cyclic voltammogram (CV) and differential pulse voltammetry (DPV) in degassed DCM with $0.1 \mathrm{M}\left[n \mathrm{Bu}_{4} \mathrm{~N}_{3} \mathrm{PF}_{6}\right.$ as the supporting electrolyte and $\mathrm{Fc} / \mathrm{Fc}^{+}$as the internal reference $\left(0.46 \mathrm{~V}\right.$ vs. $\left.\mathrm{SCE}^{27}\right)$. Absorption and steady-state PL spectra obtained in toluene at RT (SS RT), prompt PL (1-100 ns) and phosphorescence spectra (1-10 ms) obtained in toluene glass at $77 \mathrm{~K}$, measured by iCCD ( $\lambda_{\mathrm{exc}}=340 \mathrm{~nm}$ ) for (b) Cz-DiKTa, (c) Cz-Ph-DiKTa, (d) TMCz-DiKTa, (e) DMAC-DiKTa and (f) 3Cz-DiKTa.

We next evaluated the photophysical properties of the seven emitters in dropcasted 1,3bis $(N$-carbazolyl)benzene $(\mathrm{mCP})$ films at a doping concentration of $2 \mathrm{wt} \%$. This host was chosen due to its high triplet energy of $2.81 \mathrm{eV},{ }^{28}$ and the photoluminescence quantum yield $\left(\Phi_{\mathrm{PL}}\right)$ was found to be the highest at a concentration of $2 \mathrm{wt} \%$ (Tables $\mathbf{S 5}$ and $\mathbf{S 6}$ ). Indeed, $\mathbf{C z}-$ DiKTa, Cz-Ph-DiKTa, TMCz-DiKTa, DMAC-DiKTa and 3Cz-DiKTa films show high $\Phi_{\mathrm{PL}}$ values of $90 \%, 77 \%, 71 \%, 72 \%$ and $78 \%$, respectively. However, 3TMCz-DiKTa and 
3DMAC-DiKTa only present a $\Phi_{\mathrm{PL}}$ of around $20 \%$, which may be attributed to the much stronger CT band and relatively lower calculated oscillator strength for the CT states (vide supra). Further, their emission is red-shifted compared to the others, resulting in larger nonradiative decay processes. $S_{1}$ and $T_{1}$ levels in the doped film were determined from the onsets of the prompt fluorescence and phosphorescence spectra, respectively, measured at 77 $\mathrm{K}$ (Figure S11). The corresponding $\Delta E_{\mathrm{ST}}$ values of Cz-DiKTa, Cz-Ph-DiKTa and 3Cz-DiKTa are $0.14,0.10$ and $0.16 \mathrm{eV}$, respectively, which are slightly smaller than those measured in toluene glass. This may reflect changes in conformation upon slow cooling of the film in comparison to flash freezing of the toluene glass samples. DMAC-DiKTa, TMCz-DiKTa, 3TMCz-DiKTa and 3DMAC-DiKTa possess very $\Delta E_{\mathrm{ST}}$ ranging from $0.01-0.08 \mathrm{eV}$.

Table 2. Photophysical data in $2 \mathrm{wt} \%$ doped $\mathrm{mCP}$ films.

\begin{tabular}{|c|c|c|c|c|c|c|c|}
\hline Compound & $\begin{array}{l}\lambda_{\mathrm{PL}}{ }^{\mathrm{a}} \\
/ \mathrm{nm}\end{array}$ & $\begin{array}{l}\text { FWHM }^{\mathrm{b}} \\
/ \mathrm{nm}\end{array}$ & $\begin{array}{l}\Phi_{\mathrm{PL}}^{\mathrm{c}} \\
/ \%\end{array}$ & $\begin{array}{l}\tau_{\mathrm{p}}^{\mathrm{d}} ; \\
/ \mathrm{ns} ; \mu \mathrm{s}\end{array}$ & $\begin{array}{l}\mathrm{T}_{1}^{\mathrm{e}} \\
/ \mathrm{eV}\end{array}$ & $\begin{array}{l}\mathrm{S}_{1}{ }^{\mathrm{f}} \\
/ \mathrm{eV}\end{array}$ & $\begin{array}{l}\Delta E_{\mathrm{ST}}^{\mathrm{g}} \\
/ \mathrm{eV}\end{array}$ \\
\hline $\operatorname{DiKTa}^{\mathrm{f}}$ & 466 & 40 & 70 & $4.5,168$ & 2.55 & 2.75 & 0.20 \\
\hline Cz-DiKTa & 502 & 54 & 90 & $8.4,178$ & 2.48 & 2.62 & 0.14 \\
\hline Cz-Ph-DiKTa & 486 & 47 & 77 & $5.9,155$ & 2.53 & 2.63 & 0.10 \\
\hline TMCz-DiKTa & 501 & 80 & 71 & $7.6,52$ & 2.56 & 2.64 & 0.08 \\
\hline DMAc-DiKTa & 534 & 94 & 76 & $19.6,7.6$ & 2.61 & 2.57 & 0.04 \\
\hline 3Cz-DiKTa & 539 & 53 & 78 & $10.2,287$ & 2.25 & 2.41 & 0.16 \\
\hline 3TMCz-DiKTa & 577 & 110 & 18 & $22.1,3.3$ & 2.38 & 2.39 & 0.01 \\
\hline 3DMAc-DiKTa & 599 & 116 & 23 & $21.7,2.4$ & 2.32 & 2.33 & 0.01 \\
\hline
\end{tabular}

${ }^{\mathrm{a}}$ Obtained at $300 \mathrm{~K}, \lambda_{\mathrm{exc}}=340 \mathrm{~nm} ;{ }^{\mathrm{b}}$ Full-width at half-maximum; ${ }^{\mathrm{c}}$ Calculated using an integrating sphere, under $\mathrm{N}_{2}$ at $\lambda_{\mathrm{exc}}=340 \mathrm{~nm} .{ }^{\mathrm{d}}$ Measured at $\lambda_{\mathrm{exc}}=379 \mathrm{~nm}$ and $300 \mathrm{~K}$ under vacuum; ${ }^{\mathrm{f}}$ Obtained from the onset of the PL spectrum measured at $77 \mathrm{~K}$ in a time window of 1 to $100 \mathrm{~ns} ; \lambda_{\text {exc }}=343 \mathrm{~nm}$. ${ }^{\mathrm{e}}$ Obtained from the onset of the delayed spectrum $(1-10 \mathrm{~ms})$ at 77 $\mathrm{K}, \lambda_{\text {exc }}=355 \mathrm{~nm} .{ }^{\mathrm{f}}$ Obtained from the onset of the prompt spectrum $(1-100 \mathrm{~ns})$ at $77 \mathrm{~K} .{ }^{19} \mathrm{~g}$ $\Delta E_{\mathrm{ST}}=E\left(\mathrm{~S}_{1}\right)-E\left(\mathrm{~T}_{1}\right)$. 
(a)

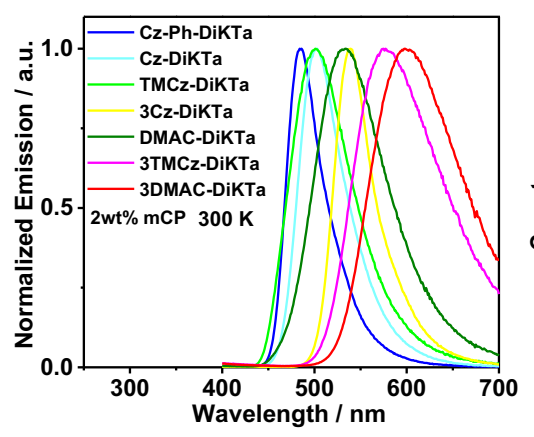

(b)

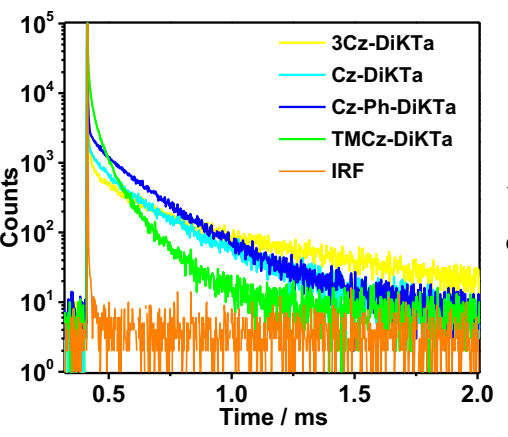

(c)

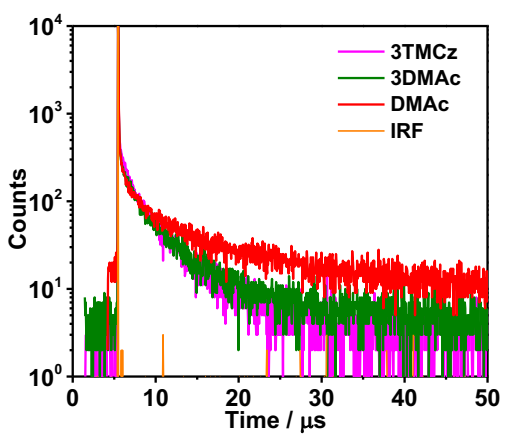

Figure 4. (a) PL spectra of Cz-DiKTa, Cz-Ph-DiKTa, 3Cz-DiKTa, TMCz-DiKTa, Cz-

DiKTa, 3TMCz-DiKTa and 3DMAC-DiKTa in $2 \mathrm{wt} \%$ doped $\mathrm{mCP}$ films at room temperature, $\lambda_{\text {exc }}=340$ nm. (b) Time-resolved PL decays of Cz-DiKTa, Cz-Ph-DiKTa, 3Cz-DiKTa and TMCz-DiKTa in $2 \mathrm{wt} \% \mathrm{mCP}$ at room temperature, $\lambda_{\mathrm{exc}}=379 \mathrm{~nm}$; (c) Time-resolved PL decays curves of DMAC-DiKTa, 3TMCz-DiKTa and 3DMAC-DiKTa in $2 \mathrm{wt} \%$ doped mCP films at room temperature and $\lambda_{\mathrm{exc}}=379 \mathrm{~nm}$.

Figures $\mathbf{4 b}$ and $\mathbf{4 c}$ show the time-resolved PL decays of the $2 \mathrm{wt} \%$ doped mCP films. All PL decays show prompt and delayed emission components at room temperature. For Cz-DiKTa, Cz-Ph-DiKTa and 3Cz-DiKTa, the prompt emission lifetimes, $\tau_{\mathrm{p}}$, are in the range of 8.4-10.2 $\mathrm{ns}$, and the delayed emission lifetimes, $\tau_{\mathrm{d}}$, are between 155-287 $\mu$ s, which are of the same order of magnitude of that of the parent compound DiKTa $(168 \mu \mathrm{s})$ in $2 \mathrm{wt} \% \mathrm{mCP}$ doped film. The compound TMCz-DiKTa shows an intermediate delayed lifetime of $52 \mu$ s, indicating that this compound possesses an excited state of intermediate character between D-A and SRCT in $\mathrm{mCP}$. By contrast, DMAC-DiKTa, 3TMCz-DiKTa and 3DMAc-DiKTa show longer prompt emission lifetimes ranging from 19.6-22.1 ns and shorter delayed emission lifetimes of 2.4-7.6 $\mu \mathrm{s}$. The shorter delayed emission lifetime of these three emitters can be attributed to their smaller $\Delta E_{\mathrm{ST}}$ values. The temperature-dependent time-resolved PL decays of all seven emitters (Figure S13) reveal the expected increase in the contribution of the delayed emission 
component with increasing temperature, which corroborates the TADF nature of these compounds.

\section{Organic light-emitting diodes}

We next fabricated vacuum-deposited OLEDs only with Cz-DiKTa, Cz-Ph-DiKTa, TMCzDiKTa, DMAC-DiKTa and 3Cz-DiKTa, as these compounds possessed suitably attractive $\Phi_{\text {PL. }}$ Here we utilized a higher concentration of emitter $(7.5 \mathrm{wt} \%)$ with the aim of improving the charge balance in the OLED device structure; we note that the photophysical behavior of the evaporated $7.5 \mathrm{wt} \%$ doped films in $\mathrm{mCP}$ is quite similar to that of the $2 \mathrm{wt} \%$ doped films in $\mathrm{mCP}$, with only a small red-shift in the emission and a small decrease in $\Phi_{\text {PL }}$ (Figures $\mathbf{S 1 5}$ and Table S7). The optimized OLED structure is shown in Figure 5a and consists of: indiumtin-oxide (ITO) / 4,4'-cyclohexylidenebis[ $N, N$-bis(4-methylphenyl)benzenamine] (TAPC, 40 $\mathrm{nm} / \mathrm{mCP}(10 \mathrm{~nm}) / \quad 7.5$ wt\% emitter:mCP $(20 \mathrm{~nm}) / 2,8$-bis(diphenyl-phosphoryl)dibenzo[b,d]thiophene, (PPT, $10 \mathrm{~nm}) /$ 1,3,5-tri(m-pyridin-3-ylphenyl)benzene (TmPyPb, 50 $\mathrm{nm}) / \mathrm{LiF}(1 \mathrm{~nm}) / \mathrm{Al}(100 \mathrm{~nm})$, where ITO acts as the transparent anode, TAPC acts as the hole transporting layer, $\mathrm{mCP}$ acts as both the electron blocking layer and the host within the emissive layer, $\mathrm{PPT}$ acts as the hole blocking layer, $\mathrm{TmPyPb}$ acts as the electron transporting layer, $\mathrm{LiF}$ acts as the electron injection layer. 
(a)

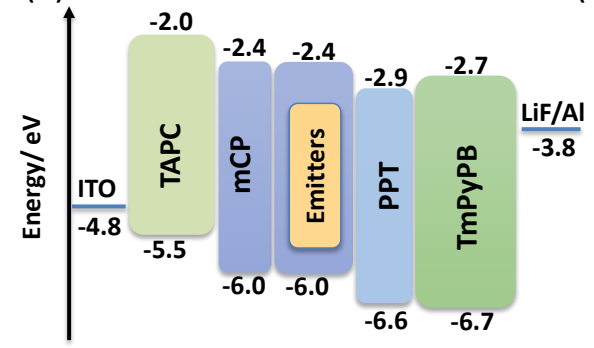

(d)

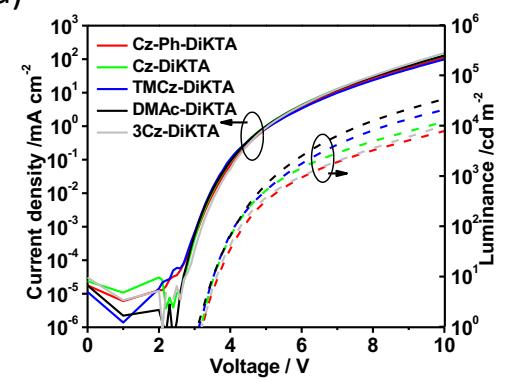

(b)

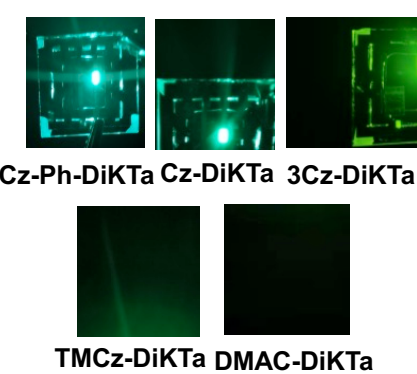

(e)

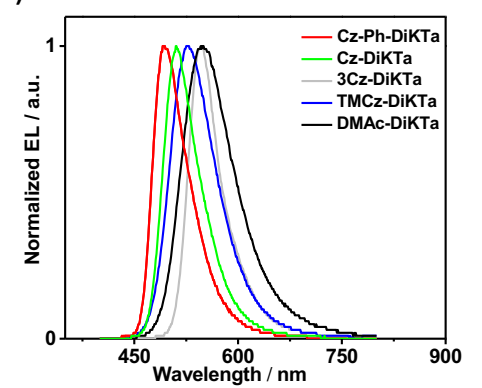

(c)

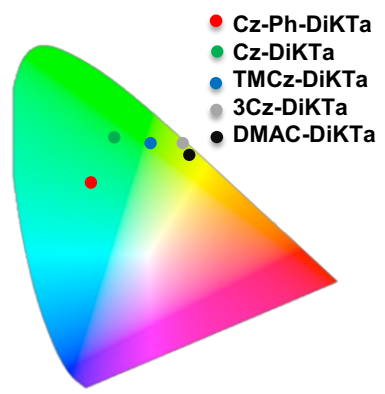

(f)

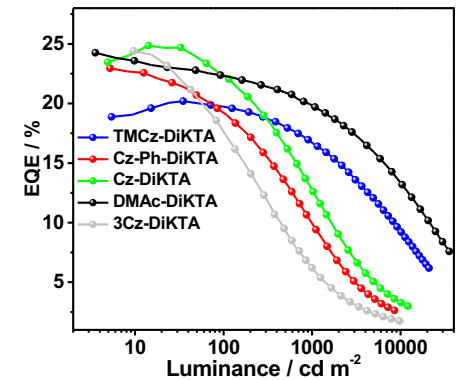

Figure 5. (a) Device configuration with energy levels and thicknesses for each layer, (b)

Photographs of OLEDs under operation. (c) Commission Internationale de L'Éclairage diagram.

(d) Current density versus voltage and luminance versus voltage curves. (e) Electroluminescence spectra. (d) External quantum efficiency (EQE) versus luminance curves.

Figure 5e illustrates the EL spectra of these devices. The OLEDs with Cz-DiKTa, Cz-Ph-

DiKTa, TMCz-DiKTa, DMAC-DiKTa and 3Cz-DiKTa show electroluminescence maxima, $\lambda_{\mathrm{EL}}$, of $511,492,527,549$, and $547 \mathrm{~nm}$, with corresponding Commission Internationale de l'Éclairage (CIE) coordinates of $(0.24,0.61),(0.18,0.50),(0.32,0.60),(0.40,0.57)$ and $(0.39$, 0.60). The devices based on the MR-TADF emitters, Cz-DiKTa, Cz-Ph-DiKTa and 3CzDiKTa, show narrow electroluminescence spectra with FWHMs of 62, 61 and $54 \mathrm{~nm}$ which are slightly broader than that of DiKTa $(39 \mathrm{~nm}) .{ }^{19}$ The FWHM values are larger for the devices with TMCz-DiKTa and DMAC-DiKTa (78 to $89 \mathrm{~nm}$ ), respectively. This reflects CT emission in these devices in line with that observed in the PL spectra. Overall, the EL emission of the devices can be tuned from sky blue to yellow green by regulating the number and the electronicdonating strength of peripheral donor around the DiKTa core.

Table 2. Summary of OLED performance 


\begin{tabular}{|c|c|c|c|c|c|c|c|}
\hline Emitters & $\begin{array}{l}\lambda_{\mathrm{EL}} \\
/ \mathrm{nm}\end{array}$ & $\begin{array}{c}\text { FWHM }^{\mathrm{a}} \\
\quad / \mathrm{nm}\end{array}$ & $\begin{array}{l}\mathrm{V}_{\text {on }}^{\mathrm{b}} \\
/ \mathrm{V}\end{array}$ & $\begin{array}{c}L_{\max }{ }^{\mathrm{c}} \\
/ \mathrm{cd} \mathrm{m}^{-2}\end{array}$ & $\begin{array}{c}\mathrm{EQE} / \%{ }^{\mathrm{d}} \\
\max / 100 / 1000\end{array}$ & $\begin{array}{l}\mathrm{PE}_{\max }^{\mathrm{e}} \\
/ \mathrm{lm} \mathrm{W}^{-1}\end{array}$ & $\mathrm{CIE}^{\mathrm{f}}$ \\
\hline Cz-DiKTa & 511 & 62 & 3.2 & 13260 & $24.9 / 20.4 / 13.0$ & 68.9 & $0.24,0.61$ \\
\hline $\begin{array}{l}\text { Cz-Ph- } \\
\text { DiKTa }\end{array}$ & 492 & 61 & 3.2 & 8529 & 23.0/19.3/10.2 & 52.3 & $0.18,0.50$ \\
\hline $\begin{array}{l}\text { TMCz- } \\
\text { DiKTa }\end{array}$ & 527 & 78 & 3.1 & 21758 & $20.2 / 19.6 / 16.7$ & 60.0 & $0.32,0.60$ \\
\hline $\begin{array}{l}\text { DMAC- } \\
\text { DiKTa }\end{array}$ & 549 & 89 & 3.1 & 35506 & 23.8/22.3/19.9 & 78.6 & $0.40,0.57$ \\
\hline $\begin{array}{c}\text { 3Cz- } \\
\text { DiKTa }\end{array}$ & 547 & 54 & 3.2 & 10796 & $24.4 / 17.3 / 6.2$ & 83.1 & $0.39,0.60$ \\
\hline
\end{tabular}

${ }^{a}$ Full width at half maximum of the EL spectrum. ${ }^{b}$ The turn-on voltage at the luminance of $1 \mathrm{~cd} \mathrm{~m}^{-2} .{ }^{\mathrm{c}}$ luminance. ${ }^{\mathrm{d}}$ Maximum external quantum efficiency/EQE at $100 \mathrm{~cd} \mathrm{~m}^{-2} / \mathrm{EQE}$ at $1000 \mathrm{~cd} \mathrm{~m}^{-2}$. ${ }^{\mathrm{e}}$ Current efficiency; ${ }^{\mathrm{f}}$ Commission Internationale de L'Éclairage coordinates.

As depicted in Figure 5f, the devices with Cz-DiKTa, Cz-Ph-DiKTa, TMCz-DiKTa, DMAcDiKTa and 3Cz-DiKTa show very high $\mathrm{EQE}_{\max }$ of $24.9 \%, 23.0 \%, 20.2 \%, 23.8 \%$ and $24.4 \%$, respectively, which is much higher than that of the DiKTa-based device with DiKTa (14.7\%) reported by our group. ${ }^{19}$ Considering the measured $\Phi_{\mathrm{PL}}$ of the doped films fabricated by thermal evaporation (see Table $\mathbf{S 7}$ ) and assuming $25 \%$ outcoupling efficiency, the $\mathrm{EQE}_{\max }$ of these devices are expected to be, respectively, $19.3 \%, 17.3 \%, 14.3 \%, 15.5 \%$ and $18.5 \%$, which are much lower than the observed $\mathrm{EQE}_{\max }$. One potential explanation would be if the transition dipole of the emitters were horizontally oriented parallel to the substrate surface, as this could lead to higher amount of the out-coupled light from the OLEDs to air. ${ }^{2}$ We therefore measured the molecular orientations of evaporated doped films of the emitters, which are identical to the ones used in the OLEDs. The angular dependent PL measurement results of these films are shown in Figure $\mathbf{S 1 8}$ and the anisotropy factors, $a$, extracted from the p-polarized emission 
were found to be 0.33 for Cz-DiKTa, 0.36 for Cz-Ph-DiKTa, 0.37 for TMCz-DiKTa, 0.36 for DMAc-DiKTa and 0.30 for 3Cz-DiKTa. These values are very close to the anisotropy factors of isotropically oriented emitters (0.33). Considering the device structure and the measured anisotropy factors, the corresponding simulated outcoupling efficiencies of these devices are, respectively, 25.4\%, 24.4\%, 23.2\%, 23.0\% and $25.4 \%$ (See SI for the details of the calculation and Table S10). Combining the measured $\Phi_{\mathrm{PL}}$ of the films and the simulated outcoupling efficiencies, the $\mathrm{EQE}_{\max }$ values were expected to be $19.6 \%, 16.8 \%, 13.1 \%, 14.3 \%$ and $18.8 \%$, respectively, which are lower than the observed values. Therefore, the emitter orientation alone cannot explain this discrepancy. Similar higher than expected performance was also found in many other MR-TADF emitters. ${ }^{22,29-30}$ Among these works, a group of structurally related emitters, QAD-Cz, QAD-2Cz and QAD-mTDPA, reported recently by Zhang and co-workers, showed near unity $\Phi_{\mathrm{PL}}$ values of $99.6 \%, 99.5 \%$ and $97.2 \%$ in $\mathrm{mCP}$ doped film, respectively, and $\mathrm{EQE}_{\max }$ of the corresponding OLEDs of $20.3 \%, 27.3 \%$ and $23.9 \%$, respectively, which implies outcoupling efficiencies of $20.4 \%, 27.4 \%$ and $27.1 \%$, respectively. ${ }^{22}$ These $\mathrm{EQE}_{\max }$ values are also higher than $20 \%$. Unfortunately, the anisotropy factors of these materials were not measured in the paper and the out-coupling efficiency was not discussed.

Although the origin of our higher-than-expected $\mathrm{EQE}_{\max }$ is not clear, we can envisage two potential causes (see "Out-coupling efficiency simulation and possible explanation about higher experimental EQE than predicted EQE" in SI). The first is that the emission efficiency of our emitters might be underestimated due to some oxygen remaining in the integrating sphere during our measurements of the $\Phi_{\mathrm{PL}}$; also, in the OLED stack, emission efficiency can be enhanced by the Purcell effect. The second potential explanation could be microcavity effects in the OLED stack leading to light emission that is directed forwards more than for a Lambertian emitter and hence increasing the apparent EQE when measured in the forward 
direction. As the main focus of this work is to demonstrate the impact of donor substitution about DiKTa-type MR-TADF compounds and how it modulates the nature of the CT character of the emitters and affects the performance of the OLEDs, the origin of the apparently high out-coupling efficiency will be investigated in future work.

In addition to the high $\mathrm{EQE}_{\max }$, these devices also show suppressed efficiency roll-off. The EQE values at $100 \mathrm{~cd} \mathrm{~m}^{-2}\left(\mathrm{EQE}_{100}\right)$ for the Cz-DiKTa, Cz-Ph-DiKTa and 3Cz-DiKTa devices are $22.5 \%, 19.2 \%, 17.8 \%$, respectively, corresponding to an efficiency roll-off of $9.6 \%, 16.5 \%$ and $27.0 \%$, respectively. This performance is improved compared to the DiKTa-based OLED ( $44 \%$ reported by us ${ }^{19}$ and $54 \%$ reported by Liao and co-workers ${ }^{29}$ ). The EQE ${ }_{1000}$ values, however, drop dramatically with efficiency roll-off of between $50 \%-74 \%$, which is not uncommon in MR-TADF-based OLEDs such as Mes ${ }_{3}$ DiKTa and the DABNA-1. ${ }^{8,}{ }^{19}$ Serious efficiency roll-off also was observed in the devices with QAD-Cz, QAD-2Cz and QADmTDPA, where the EQE dropped to $0.73 \%, 12.4 \%$ and $4.7 \%$ at $1000 \mathrm{~cd} / \mathrm{m}^{2}$, representing an efficiency roll-off greater than 55\%. By contrast, for the devices with our D-A type TADF emitters, the EQE 1000 for the OLEDs based on DMAC-DiKTa and TMCz-DiKTa are 19.9\% and $16.7 \%$, these show a much smaller efficiency roll-off of $16.4 \%$ and $17.3 \%$, which can be attributed to the smaller $\Delta E_{\mathrm{ST}}$ values. The OLED using the previously reported D-A emitter, QAO-DAd, and similar device structure also showed a comparable efficiency roll-off of $19.2 \%$ at $1000 \mathrm{~cd} / \mathrm{m}^{2}\left(\mathrm{EQE}_{\max }\right.$ of $23.9 \%$ and $\mathrm{EQE}_{1000}$ of $\left.19.3 \%\right)$. Due to the small efficiency roll-off observed in the DMAC-DiKTa device, a very high maximum brightness, $L_{\max }$, of $35500 \mathrm{~cd} \mathrm{~m}^{-}$ ${ }^{2}$ was reached (Figure $4 \mathbf{c}$ ). The device performances reported in the present study are amongst the best results in ketone-containing MR-TADF devices (and devices containing a ketonecontaining MR-TADF core as the acceptor in D-A emitters). Moreover, we demonstrate the importance of the choice of peripheral donor in order to maintain the MR-TADF character of the emitters. 


\section{Conclusions}

In summary, through attaching different numbers of donors with different electron-donating strengths at the para position to the central nitrogen atom of the previous reported DiKTa core, the character of the charge transfer excited state can be modulated from SRCT to long-range CT. This change in the nature of the emissive excited state is reflected in a broadening and a bathochromic shift of the emission. The photophysical properties, corroborated by SCS-CC2 calculations, show that the introduction of strongly electron-donating donor moieties to the periphery of the DiKTa core leads to a destabilization of the HOMO and an enhancement of the long-range CT character of the emitters. It is noteworthy that the SRCT character that is emblematic of MR-TADF compounds is conserved with the introduction of weak donors $(\mathrm{Cz}$, $\mathrm{Cz}-\mathrm{Ph}$ ) and so the color purity of these emitters is high. As a result, we achieved narrowband emission beyond $547 \mathrm{~nm}(\mathrm{FWHM}=54 \mathrm{~nm})$ in the OLED accompanied by a high $\mathrm{EQE}_{\max }$ of 24.4\% from the device with 3Cz-DiKTa. The Cz-DiKTa OLED exhibited the highest $\mathrm{EQE}_{\max }$ of $24.9 \%$ at $\lambda_{\mathrm{EL}}$ of $511 \mathrm{~nm}$. The OLED with the D-A type emitter 3DMAC-DiKTa showed high $\mathrm{EQE}_{\max }$ of $24.3 \%$ and a small roll-off of $18.5 \%$ at $1000 \mathrm{~cd} \mathrm{~m}^{-2}$. The strategy of judicioiusly decorating the MR-TADF core with weak donating groups is a useful tool to modulate the photophysical properties of these emitters and to realize high-performance OLEDs. However, too strong a choice of donor leads to the generation of donor-acceptor compounds, which leads to red-shifted and broadened emission in the device.

\section{Supporting Information}

${ }^{1} \mathrm{H}$ and ${ }^{13} \mathrm{C}$ NMR spectra, HRMS and HPLC of all target compounds; supplementary computational data; supplementary photophysical data, supplementary devices data, and orientation measurement data. 


\section{Acknowledgments}

S.W. thanks the China Scholarship Council (201906250199). EZ-C and IDWS acknowledge support from EPSRC (EP/L017008, EP/P010482/1). We are also grateful for financial support from the University of St Andrews Restarting Research Funding Scheme (SARRF) which is funded through the Scottish Funding Council grant reference SFC/AN/08/020. EZ-C is a Royal Society Leverhulme Trust Senior Research fellow (SRF\R1\201089). We would also like to thank the Leverhulme Trust (RPG-2016-047) for financial support. This project has received funding from the European Union's Horizon 2020 research and innovation programme under the Marie Skłodowska Curie grant agreement No 838885 (NarrowbandSSL). S.M.S. acknowledges support from the Marie Skłodowska-Curie Individual Fellowship. Computational resources have been provided by the Consortium des Équipements de Calcul Intensif (CÉCI), funded by the Fonds de la Recherche Scientifiques de Belgique (F.R.S.-FNRS) under Grant No. 2.5020.11, as well as the Tier-1 supercomputer of the Fédération Wallonie-Bruxelles, infrastructure funded by the Walloon Region under the grant agreement n1117545. Y.O. acknowledges funding by the Fonds de la Recherche Scientifique-FNRS under Grant n F.4534.21 (MIS-IMAGINE). D.B. is a FNRS Research Director.

\section{References}

(1) Uoyama, H.; Goushi, K.; Shizu, K.; Nomura, H.; Adachi, C. Highly efficient organic lightemitting diodes from delayed fluorescence. Nature 2012, 492 (7428), 234-8.

(2) Wong, M. Y.; Zysman-Colman, E. Purely Organic Thermally Activated Delayed Fluorescence Materials for Organic Light-Emitting Diodes. Adv Mater 2017, 29 (22), 1605444.

(3) Gibson, J.; Monkman, A. P.; Penfold, T. J. The Importance of Vibronic Coupling for Efficient Reverse Intersystem Crossing in Thermally Activated Delayed Fluorescence Molecules. ChemPhysChem 2016, 17 (19), 2956-296.

(4) Im, Y.; Kim, M.; Cho, Y. J.; Seo, J.-A.; Yook, K. S.; Lee, J. Y. Molecular Design Strategy of Organic Thermally Activated Delayed Fluorescence Emitters. Chem. Mater., 2017, 29 (5), 1946-1963, DOI: 10.1021/acs.chemmater.6b05324.

(5) Ansari, R.; Shao, W.; Yoon, S.-J.; Kim, J.; Kieffer, J. Charge Transfer as the Key Parameter Affecting the Color Purity of Thermally Activated Delayed Fluorescence Emitters. ACS ACS Appl. Mater., 2021, 13 (24), 28529-28537. 
(6) Pershin, A.; Hall, D.; Lemaur, V.; Sancho-Garcia, J. C.; Muccioli, L.; Zysman-Colman, E.; Beljonne, D.; Olivier, Y. Highly emissive excitons with reduced exchange energy in thermally activated delayed fluorescent molecules. Nat. Commun., 2019, 10 (1), 597.

(7) Madayanad Suresh, S.; Hall, D.; Beljonne, D.; Olivier, Y.; Zysman - Colman, E. Multiresonant Thermally Activated Delayed Fluorescence Emitters Based on Heteroatom Doped Nanographenes: Recent Advances and Prospects for Organic Light - Emitting Diodes. Adv. Funct. Mater., 2020, 30 (33), 201908677.

(8) Hatakeyama, T.; Shiren, K.; Nakajima, K.; Nomura, S.; Nakatsuka, S.; Kinoshita, K.; Ni, J.; Ono, Y.; Ikuta, T. Ultrapure Blue Thermally Activated Delayed Fluorescence Molecules: Efficient HOMO-LUMO Separation by the Multiple Resonance Effect. Adv Mater 2016, 28 (14), 2777-81.

(9) Yang, M.; Park, I. S.; Yasuda, T. Full-Color, Narrowband, and High-Efficiency Electroluminescence from Boron and Carbazole Embedded Polycyclic Heteroaromatics. J Am. Chem. Soc., 2020, 142 (46), 19468-19472.

(10) Zhang, Y.; Zhang, D.; Huang, T.; Gillett, A.; Liu, Y.; Cui, L.; Bin, Z.; Li, G.; Zhang, C.; Lu, Y.; Liu, Z.; Wei, J.; Duan, L. Multiple Resonance Deep-red mitters with Hybridized $\pi$ bonding/ non-bonding Orbitals to Surpass the Energy Gap Law. ChemRxiv 2020.

(11) Ikeda, N.; Oda, S.; Matsumoto, R.; Yoshioka, M.; Fukushima, D.; Yoshiura, K.; Yasuda, N.; Hatakeyama, T. Solution-Processable Pure Green Thermally Activated Delayed Fluorescence Emitter Based on the Multiple Resonance Effect. Adv. Mater., 2020, 32 (40), 2004072.

(12) Liu, G.; Sasabe, H.; Kumada, K.; Matsunaga, A.; Katagiri, H.; Kido, J. Facile synthesis of multi-resonance ultra-pure-green TADF emitters based on bridged diarylamine derivatives for efficient OLEDs with narrow emission. J. Mater. Chem. C, 2021, 9 (26), 8308-8313.

(13) Hua, T.; Zhan, L.; Li, N.; Huang, Z.; Cao, X.; Xiao, Z.; Gong, S.; Zhou, C.; Zhong, C.; Yang, C. Heavy-atom effect promotes multi-resonance thermally activated delayed fluorescence. Chem. Eng. J., 2021, 426, 131169.

(14) Zhang, Y.; Zhang, D.; Wei, J.; Liu, Z.; Lu, Y.; Duan, L. Multi-Resonance Induced Thermally Activated Delayed Fluorophores for Narrowband Green OLEDs. Angew. Chem. Int Ed. Engl., 2019, 58 (47), 16912-16917.

(15) Zhang, Y.; Zhang, D.; Wei, J.; Hong, X.; Lu, Y.; Hu, D.; Li, G.; Liu, Z.; Chen, Y.; Duan, L. Achieving Pure Green Electroluminescence with CIEy of 0.69 and EQE of 28.2\% from an Aza-Fused Multi-Resonance Emitter. Angew. Chem. Int Ed. Engl., 2020, 59 (40), 17499-17503. (16) Xu, Y.; Li, C.; Li, Z.; Wang, Q.; Cai, X.; Wei, J.; Wang, Y. Constructing Charge-Transfer Excited States Based on Frontier Molecular Orbital Engineering: Narrowband Green Electroluminescence with High Color Purity and Efficiency. Angew. Chem. Int Ed. Engl., 2020, 59 (40), 17442-17446.

(17) Qi, Y.; Ning, W.; Zou, Y.; Cao, X.; Gong, S.; Yang, C. Peripheral Decoration of MultiResonance Molecules as a Versatile Approach for Simultaneous Long-Wavelength and Narrowband Emission. Adv. Funct. Mater., 2021, 31 (29), 2102017,.

(18) Liu, Y.; Xiao, X.; Ran, Y.; Bin, Z.; You, J. Molecular design of thermally activated delayed fluorescent emitters for narrowband orange-red OLEDs boosted by a cyanofunctionalization strategy. Chem. Sci., 2021, 12 (27), 9408-9412.

(19) Hall, D.; Suresh, S. M.; dos Santos, P. L.; Duda, E.; Bagnich, S.; Pershin, A.; Rajamalli, P.; Cordes, D. B.; Slawin, A. M. Z.; Beljonne, D.; Köhler, A.; Samuel, I. D. W.; Olivier, Y.; Zysman - Colman, E. Improving Processability and Efficiency of Resonant TADF Emitters: A Design Strategy. Adv. Opt. Mater., 2019, 8 (2), 1901627.

(20) Sun, D.; Suresh, S. M.; Hall, D.; Zhang, M.; Si, C.; Cordes, D. B.; Slawin, A. M. Z.; Olivier, Y.; Zhang, X.; Zysman-Colman, E. The design of an extended multiple resonance 
TADF emitter based on a polycyclic amine/carbonyl system. Mater. Chem. Front., 2020, 4 (7), 2018-2022.

(21) Zou, S.-N.; Peng, C.-C.; Yang, S.-Y.; Qu, Y.-K.; Yu, Y.-J.; Chen, X.; Jiang, Z.-Q.; Liao, L.-S. Fully Bridged Triphenylamine Derivatives as Color-Tunable Thermally Activated Delayed Fluorescence Emitters. Org. Lett., 2021, 23 (3), 958-962.

(22) Huang, F.; Wang, K.; Shi, Y.-Z.; Fan, X.-C.; Zhang, X.; Yu, J.; Lee, C.-S.; Zhang, X.-H. Approaching Efficient and Narrow RGB Electroluminescence from D-A-Type TADF Emitters Containing an Identical Multiple Resonance Backbone as the Acceptor. ACS Appl. Mater., 2021, 13 (30), 36089-36097.

(23) Grimme, S. Density functional calculations with configuration interaction for the excited states of molecules. Chem. phys. lett., 1996, 259 (1-2), 128-137.

(24) Hirata, S.; Head-Gordon, M. Time-dependent density functional theory within the TammDancoff approximation. J. Chem. Phys., 1999, 314 (3-4), 291-299.

(25) Hall, D.; Sancho, J. C.; Pershin, A.; Beljonne, D.; Zysman-Colman, E.; Olivier, Y. The modelling of multi-resonant thermally activated delayed fluorescence emitters-properly accounting for electron correlation is key! ChemRxiv. 2021.

(26) Knöller, J. A.; Meng, G.; Wang, X.; Hall, D.; Pershin, A.; Beljonne, D.; Olivier, Y.; Laschat, S.; Zysman-Colman, E.; Wang, S. Intramolecular Borylation via Sequential B-Mes Bond Cleavage for the Divergent Synthesis of B,N,B-Doped Benzo[4]helicenes. Angew. Chem. Int Ed. Engl., 2020, 59 (8), 3156-3160.

(27) Pavlishchuk, V. V.; Addison, A. W. Conversion constants for redox potentials measured versus different reference electrodes in acetonitrile solutions at $25^{\circ} \mathrm{C}$. Inorganica Chim. Acta, 2000, 298 (1), 97-102.

(28) Bagnich, S. A.; Rudnick, A.; Schroegel, P.; Strohriegl, P.; Köhler, A. Triplet energies and excimer formation in meta- and para-linked carbazolebiphenyl matrix materials. Philos. Trans. A. Math. Phys. Eng. Sci., 2015, 373 (2044), 20140446.

(29) Yuan, Y.; Tang, X.; Du, X.-Y.; Hu, Y.; Yu, Y.-J.; Jiang, Z.-Q.; Liao, L.-S.; Lee, S.-T. The Design of Fused Amine/Carbonyl System for Efficient Thermally Activated Delayed Fluorescence: Novel Multiple Resonance Core and Electron Acceptor. Adv. Opt. Mater., 2019, 7 (7), 1801536.

(30) Han, S. H.; Jeong, J. H.; Yoo, J. W.; Lee, J. Y. Ideal blue thermally activated delayed fluorescence emission assisted by a thermally activated delayed fluorescence assistant dopant through a fast reverse intersystem crossing mediated cascade energy transfer process. J. Mater. Chem. C 201,9, 7 (10), 3082-3089.

\section{TOC}

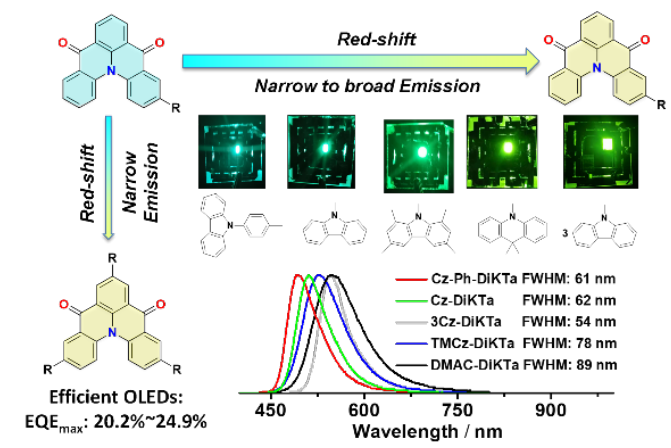

